\title{
Recent classification of diabetes mellitus
}

\author{
Available online at www.ijistweb.com \\ RESEARCH ARTICLE \\ Kuldeep Tiwari, Dinesh Kumar \\ Sri Satya Sai University of Technology \& Medical Sciences, Sehore-466001 \\ *Corresponding Author's E-mail: om11agra85@gmail.com
}

\begin{abstract}
The right classification for diabetes mellitus (DM) allows a more adequate treatment and comprises four categories: type $1 \mathrm{DM}$, type $2 \mathrm{DM}$, other types, and gestational diabetes. Assigning a type of diabetes to an individual often depends on the circumstances present at the time of diagnosis, and many diabetic individuals do not easily fit into a single class. Diabetes mellitus and lesser forms of glucose intolerance, particularly impaired glucose tolerance, can now be found in almost every population in the world and epidemiological evidence suggests that, without effective prevention and control programmes, diabetes will likely continue to increase globally. These findings were confirmed in a similar study in India, in which the FPG and 2-h PG were each strongly and equally associated with retinopathy. For both the FPG and the 2-h PG, the prevalence of retinopathy was markedly higher above the point of intersection of the two components of the bimodal frequency distribution (FPG $=129 \mathrm{mg} / \mathrm{dl}[7.2 \mathrm{mmol} / \mathrm{l}]$ and $2-\mathrm{h}$ PG $=207$ $\mathrm{mg} / \mathrm{dl}[11.5 \mathrm{mmol} / \mathrm{l}])$. In the NHANES III, 2,821 individuals aged 40-74 years received an OGTT, a measurement of $\mathrm{HbAlc}$, and an assessment of retinopathy by fundus photography.
\end{abstract}

Keywords: diabetes mellitus, diagnosis, retinopathy

\section{Introduction}

Diabetes mellitus (DM) is a group of diseases characterized by high levels of blood glucose resulting from defects in insulin production, insulin action, or both (1). The term diabetes mellitus describes a metabolic disorder of multiple aetiology characterized by chronic hyperglycaemia with disturbances of carbohydrate, fat and protein metabolism resulting from defects in insulin secretion, insulin action, or both (2).

Several pathogenic processes are involved in the development of diabetes. These range from autoimmune destruction of the $\beta$-cells of the pancreas with consequent insulin deficiency to abnormalities that result in resistance to insulin action. The effects of diabetes mellitus include long-term damage, dysfunction and failure of various organs. Diabetes mellitus may present with characteristic symptoms such as thirst, polyuria, blurring of vision, and weight loss. In its most severe forms, ketoacidosis or a non-ketotic hyperosmolar state may develop and lead to stupor, coma and, in absence of effective treatment, death (3). Often symptoms are not severe, or may be absent, and consequently hyperglycaemia sufficient to cause pathological and functional changes may be present for a long time before the diagnosis is made (4).

Symptoms of marked hyperglycemia include polyuria, polydipsia, weight loss, sometimes with polyphagia, and blurred vision. Impairment of growth and susceptibility to certain infections may also accompany chronic hyperglycemia. Acute, life-threatening consequences of uncontrolled diabetes are hyperglycemia with ketoacidosis or the nonketotic hyperosmolar syndrome. The longterm effects of diabetes mellitus include progressive development of the specific complications of retinopathy with potential blindness, nephropathy that may lead to renal failure, and/or neuropathy with risk of foot ulcers, amputation, Charcot joints, and features of autonomic dysfunction, including sexual dysfunction (5). People with diabetes are at increased risk of cardiovascular, peripheral vascular and cerebrovascular disease. Classification of diabetes mellitus is based on its aetiology and clinical presentation. As such, there are four types or classes of diabetes mellitus viz; type 1 diabetes, type 2 diabetes, gestational diabetes, and other specific types (6). 
Assigning a type of diabetes to an individual often depends on the circumstances present at the time of diagnosis, and many diabetic individuals do not easily fit into a single class. For example, a person with gestational diabetes mellitus (GDM) may continue to be hyperglycemic after delivery and may be determined to have, in fact, type 2 diabetes. It is currently a common and serious health concern globally. According to WHO, (1994), this problem has been aggravated by rapid cultural and social dynamics, ageing populations, increasing urbanization, dietary changes, reduced physical activity and other unhealthy lifestyle and behavioral patterns. Diabetes mellitus and lesser forms of glucose intolerance, particularly impaired glucose tolerance, can now be found in almost every population in the world and epidemiological evidence suggests that, without effective prevention and control programmes, diabetes will likely continue to increase globally (7).

This estimate is expected to increase to about 438 million, by 2030. Further, by 2030, the number of people with IGT is projected to increase to 472 million, or $8.4 \%$ of the adult population. The debilitating effects of diabetes mellitus include various organ failures, progressive metabolic complications such as retinopathy, nephropathy, and/or neuropathy (8). Diabetics are accompanied by risk of cardiovascular, peripheral vascular and cerebrovascular diseases (9).

\section{Materials and methods}

The methodology of the research includes the research design, description of the setting, population, sample, sampling technique and development of the tool, procedure for data collection and plan for data analysis. Efforts for improved criteria for diagnosis \& classification started a decade before Von Mering \& Minkowski's work. Lancereaux divided diabetes into the 'lean' \& 'fat' category. During pre \& post insulin eras, various adjectives were used to classify \& describe diabetes. A major requirement for epidemiological and clinical research and for the clinical management of diabetes is an appropriate system of classification that provides a framework within which to identify and differentiate it's various forms and stages.

\section{Research Design}

\section{A. Primary Data}

1] Insulin dependent diabetes mellitus (IDDM, Type 1)

2] Non-insulin dependent diabetes mellitus (NIDDM, Type 2)

a) Non obese NIDDM (Type 1 in evolution)

b) Obese NIDDM

c) Maturity onset diabetes of the young (MODY)

\section{B. Secondary:}

1] Pancreatic disease e.g- chronic pancreatitis in alcholics.

2] Hormonal abnormalities.e.g. pheochromocytoma, Cushing's syndrome.

3] Drug or chemical induced.

4] Insulin receptor abnormalities.

5] Genetic syndromes e.g. lipodystrophies.

6] Others include poorly or ill-defined types which do not fit into any of the above.

\section{Result and Discussion}

Diabetes may be diagnosed based on plasma glucose criteria, either the fasting plasma glucose (FPG) or the 2-h plasma glucose (2-h PG) value after a 75 -g oral glucose tolerance test (OGTT) or A1C criteria. (Table 4.1) FPG, 2-h PG after 75-g OGTT, and A1C are equally appropriate for diagnostic testing. It should be noted that the tests do not necessarily detect diabetes in the same individuals. The efficacy of interventions for primary prevention of type 2 diabetes has primarily been demonstrated among individuals with impaired glucose tolerance (IGT), not for individuals with isolated impaired fasting glucose (IFG) or for those with prediabetes defined by A1C criteria. The same tests may be used to screen for and diagnose diabetes and to detect individuals with prediabetes. Diabetes may be identified anywhere along the spectrum of clinical scenarios: in seemingly low-risk individuals who happen to have glucose testing, in individuals tested based on diabetes risk assessment, and in symptomatic patients. 
Table 1 Staging of type 1 diabetes

\begin{tabular}{|llll|}
\hline \multicolumn{1}{|c}{ Stage 1 } & \multicolumn{1}{c|}{ Stage 2 } & \multicolumn{1}{c|}{ Stage 3 } \\
& $\begin{array}{l}\text { Autoimmunity } \\
\text { Normoglycemia } \\
\text { Presymptomatic }\end{array}$ & $\begin{array}{l}\text { Autoimmunity } \\
\text { Dysglycemia }\end{array}$ & $\begin{array}{l}\text { New-onset hyperglycemia } \\
\text { Symptomatic }\end{array}$ \\
\hline $\begin{array}{l}\text { Diagnostic } \\
\text { criteria }\end{array}$ & Multiple & Multiple autoantibodies & Clinical symptoms \\
& autoantibodies & Dysglycemia: IFG and/or IGT & Diabetes by standard criteria \\
& No IGT or IFG & FPG 100-125 mg/dL (5.6-6.9 & \\
& & mmol/L) 2-h PG 140-199 mg/dL & \\
& & $(7.8-11.0 \mathrm{mmol} / \mathrm{L}) \mathrm{A} 1 \mathrm{C} \mathrm{5.7-}$ & \\
& & $6.4 \%(39-47 \mathrm{mmol} / \mathrm{mol})$ or & \\
& & $\$ 10 \%$ increase in A1C & \\
\hline
\end{tabular}

\section{Fasting and 2-Hour Plasma Glucose}

The FPG and 2-h PG may be used to diagnose diabetes (Table 4.2). The concordance between the FPG and 2-h PG tests is imperfect, as is the concordance between A1C and either Table 2 Criteria for the diagnosis of diabetes glucose-based test. Numerous studies have confirmed that, compared with FPG and A1C cut points, the 2-h PG value diagnoses more people with diabetes.

FPG $>126 \mathrm{mg} / \mathrm{dL}(7.0 \mathrm{mmol} / \mathrm{L})$. Fasting is defined as no caloric intake for at least $8 \mathrm{~h}$

\section{OR}

2-h PG>200 $\mathrm{mg} / \mathrm{dL}(11.1 \mathrm{mmol} / \mathrm{L})$ during an OGTT. The test should be performed as described by the WHO, using a glucose load containing the equivalent of $75 \mathrm{~g}$ anhydrous glucose dissolved in water

\section{OR}

A1C $>6.5 \%(48 \mathrm{mmol} / \mathrm{mol})$. The test should be performed in a laboratory using a method that is NGSP certified and standardized to the DCCT assay

\section{OR}

In a patient with classic symptoms of hyperglycemia or hyperglycemic crisis, a random plasma glucose $>200 \mathrm{mg} / \mathrm{dL}(11.1 \mathrm{mmol} / \mathrm{L})$.

\section{Confirming the diagnosis}

Unless there is a clear clinical diagnosis (e.g., patient in a hyperglycemic crisis or with classic symptoms of hyperglycemia and a random plasma glucose > $200 \mathrm{mg} / \mathrm{dL}$ [11.1 $\mathrm{mmol} / \mathrm{L}])$, a second test is required for confirmation. It is recommended that the same test be repeated without delay using a new blood sample for confirmation because there will be a greater likelihood of concurrence. For example, if the $\mathrm{A} 1 \mathrm{C}$ is $7.0 \%(53 \mathrm{mmol} / \mathrm{mol})$ and a repeat result is $6.8 \%(51 \mathrm{mmol} / \mathrm{mol})$, the diagnosis of diabetes is confirmed. If two different tests (such as A1C and FPG) are both above the diagnostic threshold, this also confirms the diagnosis. On the other hand, if a patient has discordant results from two different tests, then the test result that is above the diagnostic cut point should be repeated. The diagnosis is made on the basis of the confirmed test. For example, if a patient meets the diabetes criterion of the A1C (two results $\$ 6.5 \%$ [48 $\mathrm{mmol} / \mathrm{mol}])$ but not FPG (< $126 \mathrm{mg} / \mathrm{dL}[7.0 \mathrm{mmol} / \mathrm{L}])$, that person should nevertheless be considered to have diabetes.

Since all the tests have preanalytic and analytic variability, it is possible that an abnormal result (i.e., above the diagnostic threshold), when repeated, will produce a value below the diagnostic cut point. This scenario is likely for FPG and 2-h PG if the glucose samples 
remain at room temperature and are not centrifuged promptly. Because of the potential for preanalytic variability, it is critical that samples for plasma glucose be spun and separated immediately after they are drawn. If patients have test results near the margins of the diagnostic threshold, the health care professional should follow the patient closely and repeat the test in 3-6 months.

\section{Criteria for diagnosis of Diabetes Mellitus}

In 1997, the first Expert Committee on the Diagnosis and Classification of Diabetes Mellitus revised the diagnostic criteria, using the observed association between FPG levels and presence of retinopathy as the key factor with which to identify threshold glucose level.

Table 3 Criteria for testing for diabetes or prediabetes in asymptomatic adults

1. Testing should be considered in overweight or obese (BMI >25 kg/m ${ }^{2}$ or $>23 \mathrm{~kg} / \mathrm{m}^{2}$
adults who have one or more of the following risk factors:
A1C > 5.7\% ( $39 \mathrm{mmol} / \mathrm{mol})$, IGT, or IFG on previous testing
First-degree relative with diabetes
High-risk race/ethnicity (e.g., African American, Latino, Native American, Asian American,
Pacific Islander
Women who were diagnosed with GDM
History of CVD
Hypertension ( $\$ 140 / 90$ mmHg or on therapy for hypertension)
HDL cholesterol level <35 mg/dL ( 0.90 mmol/L) and/or a triglyceride level $>250 \mathrm{mg} / \mathrm{dL}(2.82$
mmol/L)
women with polycystic ovary syndrome
physical inactivity
Other clinical conditions associated with insulin resistance (e.g., severe obesity, acanthosis
nigricans).
For all patients, testing should begin at age 45 years.
If results are normal, testing should be repeated at a minimum of 3 -year intervals, with
consideration of more frequent testing depending on initial results (e.g., those with prediabetes
should be tested yearly) and risk status

\section{Genetic defects in insulin action}

There are unusual causes of diabetes that result from genetically determined abnormalities of insulin action. The metabolic abnormalities associated with mutations of the insulin receptor may range from hyperinsulinemia and modest hyperglycemia to severe diabetes. Some individuals with these mutations may have acanthosis nigricans. Women may be virilized and have enlarged, cystic ovaries. In the past, this syndrome was termed type A insulin resistance.

\section{Testing for gestational diabetes}

Previous recommendations included screening for GDM performed in all pregnancies. However, there are certain factors that place women at lower risk for the development of glucose intolerance during pregnancy, and it is likely not cost-effective to screen such patients. Pregnant women who fulfill all of these criteria need not be screened for GDM.

\section{Monogenic Diabetes Syndromes}

Monogenic defects that cause b-cell dysfunction, such as neonatal diabetes and MODY, represent a small fraction of patients with diabetes $(<5 \%)$. 
Table 4 Monogenic Diabetes

\begin{tabular}{|c|c|c|c|}
\hline & Gene & $\begin{array}{l}\text { Inheri } \\
\text { tance }\end{array}$ & Clinical features \\
\hline \multirow[t]{4}{*}{ MODY } & GCK & $\mathrm{AD}$ & $\begin{array}{l}\text { GCK-MODY: stable, nonprogressive elevated fasting } \\
\text { blood glucose; typically does not require treatment; } \\
\text { microvascular complications are rare; small rise in } 2-\mathrm{h} \\
\text { PG level on OGTT }(<54 \mathrm{mg} / \mathrm{dL}[3 \mathrm{mmol} / \mathrm{L}])\end{array}$ \\
\hline & HNF1A & $\mathrm{AD}$ & $\begin{array}{l}\text { HNF1A-MODY: progressive insulin secretory defect } \\
\text { with presentation in adolescence or early adulthood; } \\
\text { lowered renal threshold for glucosuria; large rise in } 2 \text { - } \\
\text { h PG level on OGTT (>90 } \mathrm{mg} / \mathrm{dL} \text { [ } 5 \mathrm{mmol} / \mathrm{L}] \text { ); } \\
\text { sensitive to sulfonylureas }\end{array}$ \\
\hline & HNF4A & $\mathrm{AD}$ & $\begin{array}{l}\text { HNF4A-MODY: progressive insulin secretory defect } \\
\text { with presentation in adolescence or early adulthood; } \\
\text { may have large birth weight and transient neonatal } \\
\text { hypoglycemia; sensitive to sulfonylureas }\end{array}$ \\
\hline & HNF1B & $\mathrm{AD}$ & $\begin{array}{l}\text { HNF1B-MODY: developmental renal disease } \\
\text { (typically cystic); genitourinary abnormalities; atrophy } \\
\text { of the pancreas; hyperuricemia; gout }\end{array}$ \\
\hline \multirow[t]{3}{*}{$\begin{array}{l}\text { Neonata } \\
\text { l } \\
\text { diabetes }\end{array}$} & KCNJ11 & $\mathrm{AD}$ & $\begin{array}{l}\text { Permanent or transient: IUGR; possible } \\
\text { developmental delay and seizures; responsive to } \\
\text { sulfonylureas }\end{array}$ \\
\hline & INS & $\mathrm{AD}$ & Permanent: IUGR; insulin requiring \\
\hline & & $\mathrm{AD}$ & $\begin{array}{l}\text { Transient or permanent: IUGR; rarely developmental } \\
\text { delay; responsive to sulfonylureas }\end{array}$ \\
\hline
\end{tabular}

\section{Drug- or chemical-induced diabetes}

Many drugs can impair insulin secretion. These drugs may not cause diabetes by themselves, but they may precipitate diabetes in individuals with insulin resistance. In such cases, the classification is unclear because the sequence or relative importance of $\beta$-cell dysfunction and insulin resistance is unknown.

\section{Neonatal Diabetes}

Diabetes occurring under 6 months of age is termed "neonatal" or "congenital" diabetes, and about $80-85 \%$ of cases can be found to have an underlying monogenic cause. Neonatal diabetes occurs much less often after 6 months of age, whereas autoimmune type 1 diabetes rarely occurs before 6 months of age. Neonatal diabetes can either be transient or permanent. Transient diabetes is most often due to over expression of genes on chromosome $6 \mathrm{q} 24$, is recurrent in about half of cases, and may be treatable with medications other than insulin. Permanent neonatal diabetes is most commonly due to autosomal dominant mutations in the genes encoding the Kir6.2 subunit (KCNJ11) and SUR1 subunit (ABCC8) of the b-cell $\mathrm{K}_{\mathrm{ATP}}$ channel. Correct diagnosis has critical implications because most patients with $\mathrm{K}_{\mathrm{ATP}}$ related neonatal diabetes will exhibit improved glycemic control when treated with high-dose oral sulfonylureas instead of insulin. Insulin gene (INS) mutations are the second most common cause of permanent neonatal diabetes, and, while treatment presently is intensive insulin management, there are important genetic considerations as most of the mutations that cause diabetes are dominantly inherited.

\section{Maturity-onset diabetes of the young}

MODY is frequently characterized by onset of hyperglycemia at an early age (classically before age 25 years, although diagnosis may occur at older ages). MODY is characterized by 
impaired insulin secretion with minimal or no defects in insulin action (in the absence of coexistent obesity). It is inherited in an autosomal dominant pattern with abnormalities in at least 13 genes on different chromosomes identified to date. The most commonly reported forms are GCKMODY (MODY2), HNF1AMODY (MODY3), and HNF4A-MODY (MODY1). Clinically, patients with GCKMODY exhibit mild, stable, fasting hyperglycemia and do not require antihyperglycemic therapy except sometimes during pregnancy. Patients with HNF1A- or HNF4A-MODY usually respond well to low doses of sulfonylureas, which are considered first-line therapy. Mutations or deletions in HNF1B are associated with renal cysts and uterine malformations (renal cysts and diabetes [RCAD] syndrome). Other extremely rare forms of MODY have been reported to involve other transcription factor genes including PDX1 (IPF1) and NEUROD1.

\section{Uncommon forms of immune-mediated diabetes}

In this category, there are two known conditions, and others are likely to occur. The stiff-man syndrome is an autoimmune disorder of the central nervous system characterized by stiffness of the axial muscles with painful spasms. Patients usually have high titers of the GAD autoantibodies, and approximately onethird will develop diabetes.

\section{Conclusion}

The classification of DM reflects heterogeneous nature of the condition and diverse clinical presentations. Patients with diabetic disease are characterized by insulin resistance with growing clinical and experimental evidence indicating that insulin resistance is an important and crucial player in the patho-physiology of diabetic disease.

\section{Acknowledgement}

The authors are thankful to IJIST Journal for publishing their article.

\section{Conflicts of Interest}

The author declares that there are no conflicts of interest.

\section{References}

1. Anuradha V and Vidhya D., Impact of Administration of Spirulina on Blood Glucose Levels of Selected Diabetic Patients, The Indian Journal of Nutrition and Dietetics, 2001; 38:40.

2. AOAC, Official Methods Of Analysis, The Association Of Official Analytical Chemistry, Washington, 1990.

3. Belay A., Mass Culture Of Spirulina Outdoors: The Earthrise Farms experience. In:Vonshak, A., Ed. Spirulina Platensis (Athrospira): Physiology, Cellbiology and Biotechnology, Taylor and Francis, London, 1997, Pp. 131-158.

4. Charfen M.A., Madonna F., Diabetic Ketoacidosis, Emergency Medicine Clinics of North America, 2005; 23:609-628.

5. Defronzo R. A. and Ferrannini M. D., Insulin Resistance-A Multifaceted Syndrome Responsible for NIDDM, Obesity, HTN, Dyslipidemia, Diabetes Care, 1991; 14:173-194.

6. Falquet JA, Sustainable Response to Malnutrition in Hot Regions: The Local Production of Spirulina, Antenna Technologies, Geneva, 2000.

7. Gupta A., Rajeev G., Mukesh S., Shweta R., Gupta V.P., Kunal K., Prevalence Of Diabetes, Impaired Fasting Glucose And Insulin Resistance Syndrome In An Urban Indian Population, Diabetes Research And Clinical Practice, 2003; 61:69-76.

8. Hayashi O., Katoh T., Okuwaki Y., Enhancement of Antibody Production in Mice By Dietary Spirulina Platensis, J Nutr Sci, 1994; 40:431-41.

9. Anonymous, Commercial Development of Microalgal Biotechnology: from the Test Tube to the Market Place. Biomolecular Engineering, 2003; 20(4-6):459466 